\title{
Allergen Avoidance Approaches in Food Allergy Management
}

\author{
Sibylle Koletzko, Berthold Koletzko
}

Dr. von Hauner Children's Hospital, University of Munich Medical Center, Munich, Germany

\begin{abstract}
Dietary elimination of causative food ingredients, usually food proteins, is the basis of treating food hypersensitivity. Proper diagnostic assessment is essential to avoid burdening children with unnecessary dietary restrictions with potential adverse effects. Diagnosis requires a detailed history, allergen elimination, and re-challenge with suspected foods. Complete elimination of causative food components depends on professional counseling and training of the patient and family, and transparent labeling of food products. Elimination diets carry the risk of inducing insufficient supplies of critical nutrients with adverse effects on health and wellbeing, particularly in children with exclusion of foods that provide a major part of dietary supply and patients with multiple food allergies. Infants and young children with cow's milk allergy, who have not been fully breastfed, require milk substitutes based on extensively hydrolyzed protein or amino acids. Elimination diets must be supervised and monitored to a similar degree as drug treatments, and the need for continued dietary elimination should be reviewed on a regular basis and re-challenges considered.
\end{abstract}

Copyright @ 2009 Nestec Ltd., Vevey/S. Karger AG, Basel

\section{Introduction}

Food allergy has reached an estimated prevalence of $6-8 \%$ of children affected at some time during childhood in many countries around the world with a 'Western lifestyle'. The most common causative foods are milk proteins, egg, wheat, soy, peanut and tree nuts, fish and shell fish which account for $\sim 90 \%$ of all food allergies in children. The relative frequency of reactions to various allergens differs depending on regional infant feeding patterns [1]. Allergic food reactions are also caused by a large variety of other foodstuffs. Generally, all foods containing peptides or proteins may elucidate allergic reactions. Most children react to only one or two allergens, but a small 
minority may react to several or almost all common foods (multiallergenic). These children are a particular therapeutic challenge.

Manifestation and prognosis of food allergies are modulated by the causative allergen, clinical manifestation, age at diagnosis, and underlying immunological mechanisms, all of which should be considered in management. Most children with cow's milk protein (CMP) allergy (CMPA) or hen's egg allergy become tolerant by the age of 3-5 years [2], but allergies against peanuts, fish and shellfish often persist. Children with positive specific IgE antibodies against causative food at diagnosis tend to develop tolerance to these food antigens later than those negative for specific antibodies or skin prick test reactions [2].

Treating food allergy and other food intolerances is based on dietary elimination of causative food ingredients, usually food proteins. When a therapeutic elimination diet is introduced, the focus of the patient, the patient's family and healthcare providers is on the rapid and possibly complete elimination of symptoms, but any therapeutic diet must also adequately nourish the patient, i.e. it must provide all nutrients needed for growth, normal development and performance, and it should avoid untoward effects as much as possible. In infants and young children it is a particular challenge to meet all the dietary requirements with a restricted diet as the nutrient needs per unit body weight are particularly high at a young age due to the added needs for growth.

Of particular concern are restrictive elimination diets without appropriate counseling, and in children those based on complementary diagnostic allergy testing are of unproven diagnostic value [3]. Food allergy is often misconceived. For example, the self-reported lifetime prevalence of adverse food reactions was 34.9\% in over 4,000 German persons (mean age 41 years) [4], of whom 20\% altered their diet because of perceived adverse reactions to food. Noimark and Cox [5] reported a 5-year-old boy with mild atopic eczema who developed failure to thrive due to exclusion of a variety of foods including dairy products, eggs, gluten, beef, chicken, fish, citrus, tomatoes and strawberries, after evaluation by a practitioner following naturopathy concepts. The reported hazards of elimination diets in children include rickets, kwashiorkor, and vitamin and mineral deficiencies. Therefore, it is essential that an accurate diagnosis of food allergy is made by a physician with appropriate training, and that allergen elimination diets are supervised by experienced pediatric dieticians.

\section{Allergen Avoidance for Diagnostic Evaluation}

Successful management of food allergy depends on identifying the offending foods or antigens and complete elimination. An accurate patient history is essential but not always sufficient to identify causative foods. Diagnostic elimination of suspected food antigens and a controlled food challenge, 
preferentially in a double-blind fashion, is considered the gold standard for diagnosing food allergy [6]. The required duration of diagnostic elimination diets depends on the symptoms: for acute reactions like urticaria or immediate vomiting a few days are sufficient until symptoms have resolved and the challenge can be performed. For some gastrointestinal manifestations like dysmotility, allergic gastritis or enterocolitis, or for atopic dermatitis with more fluctuating symptoms, an elimination of 2 weeks or even longer is recommended.

Diagnostic elimination diets comprise: (1) elimination of one or several suspected food items; (2) elimination of all but a defined group of 'allowed' foods (oligoantigenic or 'few food' diet), and (3) formulae based on extensive hydrolyzed protein or amino acids.

While the first approach is preferred if causative foods are identified by a clear history or allergy tests, the other two options are necessary if no specific food is suspected, symptoms are unspecific and/or multiple food allergy is suspected. For the first two approaches and complete elimination of the allergens, adequate information must be provided to the parents and children (appropriate for their age and understanding) about the allergen, the uses of synonymous terms (for example, wheat and gluten or semolina, or milk and CMP, and whey and casein), and the types of food in which it may be found.

Oligoantigenic or 'few food' diets consist of one meat (turkey or lamb), one carbohydrate source (potato or rice) and one fruit (pear or banana), and two vegetables (cauliflower, broccoli, carrots, cabbage, or parsnips) plus water. Oligoantigenic diets should be followed no longer than 2-3 weeks [7] because they may result in caloric deficiency due to poor palatability, or in deficiencies of macro- or micronutrients due to an imbalanced diet.

\section{Food Challenges}

If symptoms resolve or improve during the elimination diet, food allergy should be confirmed by a controlled food challenge supervised by a physician [8]. If several foods are eliminated, these foods should be re-introduced one by one to identify the causative foods. In infants and very young children an open or single-blind challenge may be sufficient. In case of doubt and in children suffering from subjective symptoms like abdominal pain or nausea, results should be confirmed by double-blind placebo-controlled challenge, but false-positive and negative reactions may occur even in double-blind controlled food challenges [9].

Prolonged elimination diets are only justified after diagnosis is proven by a positive food challenge, and not only based on positive results of specific IgE testing or atopy patch test. If symptoms do not improve on antigen elimination or do not exacerbate after challenge, the diet should be discontinued. In children with atopic dermatitis unnecessary elimination diets are very com- 
Table 1. Risks and disadvantages of the different diagnostic or therapeutic diets, graded from missing $(-)$ to strongly present $(+++)$

Elimination of one Oligoantigenic diet Extensive hydrolyzed or few foods or amino acid-based formula

\begin{tabular}{llcl}
\hline $\begin{array}{l}\text { Risk of nutritional } \\
\text { deficits }\end{array}$ & $-/+$ & +++ & $-1+$ \\
$\begin{array}{l}\text { Not palatable or } \\
\text { accepted }\end{array}$ & - & ++ & $\begin{array}{l}+++++ \\
\text { (depends on age) }\end{array}$ \\
$\begin{array}{l}\text { Psychological or } \\
\text { social problems }\end{array}$ & - & ++ & ++++ \\
$\begin{array}{l}\text { Incomplete } \\
\text { elimination of }\end{array}$ & ++ & ++ & - \\
$\begin{array}{l}\text { antigen } \\
\text { Costs }\end{array}$ & & & \\
\hline
\end{tabular}

mon, particularly cows' milk-free diets. Sinagra et al. [10] studied 186 Italian children (aged 4-69 months) with atopic dermatitis for specific IgE, atopy patch test and oral milk protein provocation, and confirmed CMPA in only $4 \%$ in contrast to $24 \%$ who followed a CMP-free diet.

\section{Allergen Avoidance for Treatment of Confirmed Food Allergy}

Elimination diets remain effective and are usually the only treatment for food allergy, Before counseling children and caregivers one must consider how strict the diet must be. In case of non-IgE-mediated reactions with mild symptoms, e.g. constipation, mild atopic dermatitis, or reactions to only large doses, precautions are less important than in children with life-threatening reactions to very small doses. In these children oral tolerance induction has been attempted with some success, but complete tolerance is not achieved [11]. It is also unresolved whether complete and prolonged elimination may increase the risk of a severe acute reaction and reduces the chance of tolerance induction [12], or whether complete elimination leads to an earlier loss of allergy.

While the short duration of a diagnostic elimination diet is less likely to cause nutritional deficits or negative psychosocial effects, longer elimination diets may cause serious problems (table 1). Elimination of one food or several foods is easy if the food is not commonly consumed (e.g. shell fish or kiwi). However, elimination of foods contributing a major part to the nutrient supply (e.g. cow's milk-based products) may put the child at significant risk, e.g. an inadequate calcium intake, low bone density, and rickets. Some causative 
Table 2. Unexpected food sources of common allergens

\begin{tabular}{ll}
\hline Food & Ingredient \\
\hline Soy sauce & Wheat \\
Malt sugar, corn flakes & Wheat, barley \\
Low-fat peanut butter & Soy protein \\
Low-fat camembert & Wheat \\
Sweet and sour sauce & Wheat or soy \\
Ham & Milk protein \\
Egg substitutes & Egg white \\
Hamburger & Soy protein \\
Ham, cold cuts & Wheat and milk protein \\
\hline
\end{tabular}

antigens are hidden in products, e.g. soy or wheat proteins (table 2). For some antigens (e.g. peanuts, shell fish) only traces or inhalation may cause reactions. Food preparation may influence the severity of reactions; cooking reduces the antigenicity of most fruits and vegetables, but the antigenicity of peanuts is increased by roasting.

\section{Risk of Malnutrition}

Adverse effects of elimination diets on nutrient supply and child growth have been reported. Laitinen et al. [13] in Finland followed 159 children from birth to 4 years to evaluate a probiotic intervention. Children who developed atopic eczema had significantly reduced intakes of protein, vitamin A and calcium at different time points of the study and grew less well then children without atopy. Children with atopic dermatitis had 5.1\% lower weight for height ( $95 \%$ CI -8.9 to $-1.2 ; \mathrm{p}=0.01$ ) than controls and were significantly shorter at 2 years (height SD score $0.4,95 \%$ CI $0.17-0.65)$ than controls $(0.73$, 95\% CI 0.51-0.96; $\mathrm{p}=0.048$ ).

Fox et al. [14] reported on a 14-month-old boy who had been exclusively breastfed until 5 months when complementary feeding based on rice and vegetables was introduced. A cow's milk formula caused immediate generalized urticaria at 6 months, and a similar reaction occurred to goat's milk-based formula, and atopic eczema developed. The mother continued breastfeeding until 14 months and provided selected complimentary foods including vegetables and fruits, but avoided eggs, fish and CMP. At 14 months, the child was severely malnourished (weight and height $<3$ rd centile) with iron deficiency anemia and vitamin D deficiency rickets. Thus, dietary modification in atopic infants and young children may induce severe adverse effects on nutrient supply, growth and health, and hence appropriate and qualified guidance is required when using elimination diets. 
Dietary exclusion of fish and seafood results in a near total elimination of the n-3 long-chain polyunsaturated fatty acid (PUFA), docosahexaenoic acid (DHA). We estimated that a 6-year-old child consuming a mixed diet with regular consumption of fatty fish (e.g. salmon) achieves a dietary DHA intake of about $640 \mathrm{mg} /$ day, whereas the elimination of all fish and seafood would reduce the dietary DHA intake more than 30-fold to no more than 20 $\mathrm{mg} /$ day [15]. This is of concern given the indications of the importance of a dietary DHA supply for visual and neuromotor functions in infancy and childhood. Children with elimination of fish and seafood from their diet should preferably receive an alternative source of long-chain n-3 long-chain PUFA. Most children with allergic reactions to seafood easily tolerate purified fish oils, because they are devoid of appreciable residues of fish protein, but it is prudent to supply the first dose under medical supervision and not to change the source without controlled exposure. Alternatives are DHA-rich single-cell oils from fermented algal sources that do not contain fish or seafood proteins. The supply of DHA-rich oils may be complemented by foods and oils providing the n-3 fatty acid precursor $\alpha$-linolenic acid, such as rapeseed oils, walnut oil, soybean oil, and walnuts, since over time even the limited activity of conversion of $\alpha$-linolenic acid will yield some DHA.

The most frequently eliminated food allergen from the diet of infants and children is CMP. Compared to children on unrestricted diets, elimination of all foods containing CMP will usually lead to a major change in nutrient supply, with potential consequences for long-term health. Medeiros et al. [16] compared the food intake and nutritional status of 26 children (mean age 19 months) on a diet free of cow's milk and cow's milk products to 30 children (mean age 17 months) with unrestricted diets. Children on a CMP-free diet had lower dietary intakes of energy $(p=0.005)$, protein $(p<0.001)$, fat ( $p$ $<0.001)$, calcium $(\mathrm{p}<0.001)$ and phosphorous $(\mathrm{p}<0.001)$ than controls, and lower standard deviation scores (z scores) for height for age $(-0.81 \pm$ 0.06 vs. $+0.42 \pm 0.25, \mathrm{p}<0.001)$, weight for age $(-1.03 \pm 1.21$ vs. $+0.02 \pm$ $0.91, \mathrm{p}<0.001)$, and weight for height $(-0.63 \pm 1.08$ vs. $+0.30 \pm 1.11, \mathrm{p}=$ 0.004). Moreover, unless implementing appropriate precautions, a low intake of calcium and phosphorus resulting from elimination of all cow's milk-based products will induce reduced bone mineralization and a decreased peak bone mass, with short- and long-term adverse consequences for fracture risk.

These and other observations indicate that strict dietary elimination without adequate precautionary measures may adversely affect substrate intake, child growth and long-term health and wellbeing. Therefore, elimination diets should only be introduced and maintained if one can expect a clear benefit. The continued need for elimination diets must be reexamined over time, and repeated food challenges be considered. If an elimination diet appears indicated and necessary, qualified dietary counseling and regular monitoring of growth are essential. Guided by the situation of the individual patient and its dietary characteristics, specific markers should be assessed 
such as plasma concentrations of vitamin D or n-3 PUFAs, or measurements of bone density.

\section{Incomplete Allergen Elimination due to Contamination or Insufficient Labeling}

Elimination diets carry the risk of incomplete elimination due to hidden antigens, cross-reactivity, insufficient labeling or contamination. Contamination may occur at different stages of food processing or packing. This is a particular problem for grains which grow next to each other on fields, may be harvested and transported with the same machines and vehicles, and be ground in the same mill. For examples, commercial oat products very often contain wheat protein (gluten). Even within one and the same brand of oat products, a wide batch-to-batch variation in the contaminated antigens may occur from 1,800 to $<3 \mathrm{mg} / \mathrm{kg}$ [17]. Therefore, commercial oat products cannot be recommended in wheat allergy unless manufacturers certify products as wheat/ gluten-free. Another common example is the occurrence of peanut allergen in margarine or whole milk chocolate. The risk of allergen contamination for an allergic reaction or even life-threatening event depends on the threshold dose of an offending food eliciting adverse reactions [18] which differs between individuals, depends on food processing and the type of allergic reaction. Sicherer et al. [19] reported the dose response in double-blind, placebo-controlled oral food challenges in 196 children with atopic dermatitis and known food allergy against egg, milk, soy, wheat, peanut or fish. About half of the children with milk and egg allergy reacted to a dose of $500 \mathrm{mg}$ or less, some even to $100 \mathrm{mg}$. Neither the serum concentration of food-specific IgE nor the absolute wheal size on skin prick test correlated with the dose causing a reaction or the severity of the reaction. Thus, even very small amounts of residual or contaminant allergens in food products may put a highly sensitized child at risk.

Correct and complete labeling of food products for frequent allergens is important to protect the health and wellbeing of allergic individuals. Legislation in the European Union requires clear labeling of any content of 20 common food antigens on all food products, even if they occur in only small quantities. Patients and caregivers should be instructed to read product labels each time before use because ingredients may change without warning.

\section{Cross-Reactivity of Food Antigens}

Cross-reactivity of allergens due to reactions toward shared homologous proteins may be the cause of reactions to a variety of related foods of plant or animal origin. About $90 \%$ of patients with CMPA also reacted to goat's milk in 
an Italian study; hence goat's milk generally is not considered to be a suitable alternative to cow's milk or to cow's milk-based infant formula [20]. Milk from other animals like mares or camels might be safer, but these milks are not easily available and also are not nutritionally adequate to meet the substrate needs of infants. Some patients with allergy to mammalian milks also react to mammalian meats due to homologous proteins. Werfel et al. [21] reported beef allergy in $9.7 \%$ of 62 children with proven CMPA. Cross-reactivity between egg and avian meat occurs, particularly chicken meat allergy in patients with hen's egg allergy. Children with allergic reactions to chicken meat may also react to other poultry such as turkey or pheasant. In fish-allergic patients reactions to more than one type of fish are common.

Sensitization to several allergenic plant foods (e.g. peanut, tree nuts, sesame or poppy seed) is common. Reactions to peanut and tree nut (hazelnut and/or Brazil nut) were reported in 449 (59\%) of 731 patients with peanut allergy [22]. Wheat, rye, barley, and to a lesser extent oat, share homologous proteins. Patients with wheat allergy often show co-sensitization to these different grains.

\section{Breast Milk Substitutes for Infants with Cow's Milk Protein Allergy}

Food-allergic infants need a suitable breast milk substitute that provides all nutrient needs during the critical period of growth and development.

Unmodified mammalian milks, including unmodified cow's, sheep, buffalo, mare or goat's milk, and unmodified soy or rice milk, are contraindicated since their composition is unsuitable to meet the metabolic requirements of human infants, and possible allergenic cross-reactivity poses additional risks.

Infant formulae based on soy protein have widely been used in infants with CMPA because of the lower cost and often better taste and acceptance as compared to therapeutic formulae, but soy allergies are frequently observed in CMPA patients [23]. Allergic manifestations to soy formula in infants at risk of atopy is comparably higher than to cow's milk formula [24]. Intolerance to soy has been reported in 10-14\% [25, 26] of infants with CMPA, whether positive or negative for specific IgE antibodies to CMP [25]. The risk increased to $25 \%$ if soy formula was introduced during the first 6 months [25]. Nutritional aspects of soy protein formulae have recently been reviewed by the ESPGHAN Committee on Nutrition [27]. Soy formulae support normal growth and nutrient status [28], but soy protein is inferior to CMP in its biological value for infants, with a lower digestibility and bioavailability of the protein and with lower methionine content [27]. Accordingly, European Union legislation on infant formulae requires a higher minimum protein content in soy formulae than CMP formulae (2.25 vs. $1.8 \mathrm{~g} / 100 \mathrm{kcal}$ ). High concentrations of aluminum and phytoestrogens (isoflavones) are found in soy protein formulae, the 
long-term effects of which are unknown. ESPGHAN recommended that soy protein formulae should not be used in infants with food allergy during the first 6 months of life. If soy protein formulae is considered in food-allergic infants after the age of 6 months because of their lower cost and better acceptance relative to extensive protein hydrolysate formulae, tolerance to soy protein should first be established by clinical challenge in the individual patient [27]. The conclusions of ESPGHAN were largely shared by the Committee on Nutrition of the American Academy of Pediatrics in their recent statement that extensively hydrolyzed formulae, but not soy formula, should be given to infants with proven cow's milk allergy [29].

The dietary treatment of choice for infants with CMPA is a therapeutic hypoallergenic formula based on extensively hydrolyzed protein, or on amino acid mixtures. Therapeutic formulae for CMPA must be tolerated by at least 90\% (with 95\% confidence) of CMPA infants. To demonstrate this, a minimum of 29 patients with CMPA must be enrolled in a study documenting tolerance. If none of the 29 infants with proven CMPA reacts, then there is $95 \%$ certainty that $90 \%$ of allergic individuals will not react to that formula [30]. These criteria are met by some extensively hydrolyzed formulae based on whey, casein or other protein sources, and by amino acid-based formulae, whereas formulae based on mildly hydrolyzed proteins used for allergy prevention are generally unsuitable for treatment because of the residual allergen content [31]. Extensively hydrolyzed formulae are the first choice in infants with mild to moderate CMPA for reasons of cost, although in some cases the cost of extensively hydrolyzed formulae and amino acid-based formulae may be comparable [32]. However, allergic reactions even to extensively hydrolyzed therapeutic formulae are possible [33, 34]. Residual allergens in extensively hydrolyzed formulae appear more likely to produce gastrointestinal and other non-IgE-associated manifestations [35, 36], but IgE-related reactions to extensive hydrolyzed formulae have also been reported [35]. In such infants a switch to another type of extensively hydrolyzed formula or to an amino acidbased formula may be tried. Amino acid-based formulae are also indicated in infants or young children with a history of life-threatening (respiratory or anaphylactic) reactions, or in those who refuse extensively hydrolyzed formulae which tend to have a more bitter taste than amino acid-based formulae.

Therapeutic hypoallergenic formulae based on extensively hydrolyzed proteins or on amino acid mixtures can generally be regarded as effective and safe, and as supporting adequate growth. However, the properties of various products differ, and the biological value of the nitrogen sources in extensively hydrolyzed proteins or amino acid mixtures for infants appears not be equal to that of CMP. Maggio et al. [37] compared the effects of formulae based on hydrolyzed CMPs and on intact CMPs in preterm infants in a randomized controlled trial. At equal daily feeding volumes, nitrogen and energy intakes, infants fed hydrolysates had less weight gain (17.4 \pm 3.4 vs. $20.5 \pm 3.3 \mathrm{~g} / \mathrm{kg} /$ day; $\mathrm{p}=0.045$ ) and lower change in standard deviation scores (z scores) for 
weight $(-0.18 \pm 0.16$ vs. $0.00 \pm 0.09 ; \mathrm{p}=0.009)$ and/or head circumference $(-0.06 \pm 0.13$ vs. $0.06 \pm 0.13 ; p=0.049)$. After 2 weeks, infants receiving hydrolyzed formula also had higher urinary levels of essential amino acids. These data point to a lesser nutritional value of hydrolyzed than intact proteins for infants.

In term infants, Rigo et al. [38] compared growth parameters, biochemical indices of protein metabolism and plasma amino acid concentrations in infants fed either human milk or 5 different hydrolyzed formulae during the first month of life. While growth parameters and the various protein concentrations determined were similar in whey hydrolysate formulae to those observed with human milk, the other hydrolysates led to a lesser gain in weight and in other growth parameters. Biochemical parameters, such as blood urea nitrogen, serum total protein, transferrin and plasma amino acids, differed between the groups fed different hydrolysates.

In a controlled study from 6 weeks to 6 months of age, term infants were breastfed or fed regular formula based on intact CMP, two different casein hydrolysates, or a whey hydrolysate [39]. Infants fed the casein hydrolysate formulae had significantly lower serum ferritin and significantly higher serum urea nitrogen values than the other groups. In all hydrolysate formula groups, the plasma amino acids, threonine, valine, phenylalanine, methionine, and tryptophan, were significantly higher than in the breastfed control group. Individual amino acid concentrations differed between the different hydrolysate groups. The authors concluded that the amounts of amino acids provided by hydrolyzed formulae is excessive compared with regular formula, which is reflected by the high serum urea nitrogen and plasma amino acid concentrations, and they suggested that a reduced and more balanced amino acid content of hydrolyzed formulae may be beneficial.

\section{Conclusions and Outlook}

Proper diagnostic assessment is essential for the diagnosis of food allergy. Elimination diets should only be introduced and maintained if one can expect a clear benefit. Antigen elimination with improvement in symptoms and food challenge with recurrence of symptoms still remains the gold standard for food hypersensitivity. Effective treatment with complete elimination requires good counseling and training, and transparent labeling of food product components. Infants and young children need breast milk, and if not available or tolerated, a milk substitute with extensively hydrolyzed protein or amino acid-based formula should be supplied. The continued need for elimination diets must be reexamined over time, and repeated food challenges be considered. Further research is needed to assess the best strategies to assume tolerance as early as possible. With adequate treatment, a good outcome and good quality of life will be a achieved for the child and its family. 


\section{References}

1 Chiang WC, Kidon MI, Liew WK, et al: The changing face of food hypersensitivity in an Asian community. Clin Exp Allergy 2007;37:1055-1061.

-2 Host A, Halken S, Jacobsen HP, et al: Clinical course of cow's milk protein allergy/intolerance and atopic diseases in childhood. Pediatr Allergy Immunol 2002;13(suppl 15):23-28.

- 3 Niggemann B, Gruber C: Unproven diagnostic procedures in IgE-mediated allergic diseases. Allergy 2004;59:806-808.

4 Zuberbier T, Edenharter G, Worm M, et al: Prevalence of adverse reactions to food in Germany - a population study. Allergy 2004;59:338-345.

-5 Noimark L, Cox HE: Nutritional problems related to food allergy in childhood. Pediatr Allergy Immunol 2008;19:188-195.

-6 Gellerstedt M, Bengtsson U, Niggemann B: Methodological issues in the diagnostic work-up of food allergy: a real challenge. J Investig Allergol Clin Immunol 2007;17:350-356.

7 Grimshaw KE: Dietary management of food allergy in children. Proc Nutr Soc 2006;65:412417.

8 Niggemann B, Beyer K: Diagnosis of food allergy in children: toward a standardization of food challenge. J Pediatr Gastroenterol Nutr 2007;45:399-404.

-9 Niggemann B, Beyer K: Pitfalls in double-blind, placebo-controlled oral food challenges. Allergy 2007;62:729-732.

-10 Sinagra JL, Bordignon V, Ferraro C, et al: Unnecessary milk elimination diets in children with atopic dermatitis. Pediatr Dermatol 2007;24:1-6.

-11 Staden U, Rolinck-Werninghaus C, Brewe F, et al: Specific oral tolerance induction in food allergy in children: efficacy and clinical patterns of reaction. Allergy 2007;62:1261-1269.

-12 Flinterman AE, Knulst AC, Meijer Y, et al: Acute allergic reactions in children with AEDS after prolonged cow's milk elimination diets. Allergy 2006;61:370-374.

13 Laitinen K, Kalliomaki M, Poussa T, et al: Evaluation of diet and growth in children with and without atopic eczema: follow-up study from birth to 4 years. Br J Nutr 2005;94:565-574.

14 Fox AT, Du-Toit G, Lang A, Lack G: Food allergy as a risk factor for nutritional rickets. Pediatr Allergy Immunol 2004;15:566-569.

15 Koletzko B: Nutritional considerations on elimination diets and on substitutes for human milk and cows' milk based infant formulae; in Koletzko S (ed): Food Allergy in Childhood. Causes and Consequences. Heilbronn, SPS, 2007, pp 158-168.

16 Medeiros LC, Speridião PG, Sdepanian VL, et al: Nutrient intake and nutritional status of children following a diet free from cow's milk and cow's milk by-products (in Portugese). J Pediatr (Rio J) 2004;80:363-370.

17 Thompson T: Gluten contamination of commercial oat products in the United States. N Engl J Med 2004;351:2021-2022.

-18 Taylor SL, Hefle SL, Bindslev-Jensen C, et al: A consensus protocol for the determination of the threshold doses for allergenic foods: how much is too much? Clin Exp Allergy 2004;34:689-695.

-19 Sicherer SH, Morrow EH, Sampson HA: Dose-response in double-blind, placebo-controlled oral food challenges in children with atopic dermatitis. J Allergy Clin Immunol 2000;105:582586.

20 Bellioni-Businco B, Paganelli R, Lucenti P, et al: Allergenicity of goat's milk in children with cow's milk allergy. J Allergy Clin Immunol 1999;103:1191-1194.

21 Werfel SJ, Cooke SK, Sampson HA: Clinical reactivity to beef in children allergic to cow's milk. J Allergy Clin Immunol 1997;99:293-300.

-22 Pumphrey R, Wilson P, Faragher E, Edwards S: Specific immunoglobulin E to peanut, hazelnut and brazil nut in731 patients: similar patterns found for all ages. Clin Exp Allergy 1999;29:1256-1259.

23 Host A, Koletzko B, Dreborg S, et al: Dietary products used in infants for treatment and prevention of food allergy. Joint Statement of the European Society for Paediatric Allergology and Clinical Immunology (ESPACI) Committee on Hypoallergenic Formulas and the European Society for Paediatric Gastroenterology, Hepatology and Nutrition (ESPGHAN) Committee on Nutrition. Arch Dis Child 1999;81:80-84.

24 Osborn DA, Sinn J: Soy formula for prevention of allergy and food intolerance in infants. Cochrane Database Syst Rev 2004;3:CD003741. 
25 Klemola T, Vanto T, Juntunen-Backman K, et al: Allergy to soy formula and to extensively hydrolyzed whey formula in infants with cow's milk allergy: a prospective, randomized study with a follow-up to the age of 2 years. J Pediatr 2002;140:219-224.

-26 Zeiger RS, Sampson HA, Bock SA, et al: Soy allergy in infants and children with IgE-associated cow's milk allergy. J Pediatr 1999;134:614-622.

-27 Agostoni C, Axelsson IE, Goulet O, et al: Soy protein infant formulae and follow-on formulae: a commentary by the ESPGHAN Committee on Nutrition. J Pediatr Gastroenterol Nutr 2006;42:352-361.

-28 Seppo L, Korpela R, Lönnerdal B, et al: A follow-up study of nutrient intake, nutritional status, and growth in infants with cow milk allergy fed either a soy formula or an extensively hydrolyzed whey formula. Am J Clin Nutr 2005;82:140-145.

-29 Bhatia J, Greer F: Use of soy protein-based formulas in infant feeding. Pediatrics 2008; 121:1062-1068.

30 Koletzko S: Food allergen avoidance for treatment and prevention; in Koletzko S (ed): Food Allergy in Childhood. Causes and Consequences. Heilbronn, SPS, 2007, pp 190-211.

31 Koletzko S: Allergy prevention through early nutrition; in Koletzko B, Cooper P, Makrides M, et al (eds): Pediatric Nutrition in Practice. Basel, Karger, 2008, pp 106-109.

-32 Niggemann B, von Berg A, Bollrath C, et al: Safety and efficacy of a new extensively hydrolyzed formula for infants with cow's milk protein allergy. Pediatr Allergy Immunol 2008;19:348354.

-33 Wahn U, Wahl R, Rugo E: Comparison of the residual allergenic activity of six different hydrolyzed protein formulas. J Pediatr 1992;121:S80-S84.

34 Rosendal A, Barkholt V: Detection of potentially allergenic material in 12 hydrolyzed milk formulas. J Dairy Sci 2000;83:2200-2210.

-35 Sicherer SH, Noone SA, Koerner CB, et al: Hypoallergenicity and efficacy of an amino acidbased formula in children with cow's milk and multiple food hypersensitivities. J Pediatr 2001;138:688-693.

-36 de Boissieu D, Dupont C: Allergy to extensively hydrolysed cows' milk proteins in infants: safety and duration of amino acid-based formula. J Pediatr 2002;141:271-273.

-37 Maggio L, Zuppa AA, Sawatzki G, et al: Higher urinary excretion of essential amino acids in preterm infants fed protein hydrolysates. Acta Paediatr 2005;94:75-84.

38 Rigo J, Salle BL, Putet G, Senterre J: Nutritional evaluation of various protein hydrolysate formulae in term infants during the first month of life. Acta Paediatr Suppl 1994;402:100-104.

-39 Hernell O, Lönnerdal B: Nutritional evaluation of protein hydrolysate formulas in healthy term infants: plasma amino acids, hematology, and trace elements. Am J Clin Nutr 2003;78:296301 .

\section{Discussion}

Dr. Isolauri: I would like to make one comment on better growth with one formula compared to the other. In children who have early onset cow's milk allergy there is frequently failure to thrive especially if they have gastrointestinal (GI) symptoms, and this is due to local inflammation and by putting them on elimination diets. The sooner the inflammation stops the better they grow. So better growth in these children is not simply that they grow bigger but they have faster catch-up growth and their clinical problems resolve sooner. Do you agree with this or do you want to clarify what you mean by better growth?

Dr. S. Koletzko: No, I think you have to distinguish between therapy and prevention. If you look at therapy of course, many of them have failure to thrive due to GI involvement even if it is not obvious. You have shown that they have loss of albumin and mild enteropathy, many of them with atopic dermatitis. So I think treatment and catch-up growth are very important, but the difference is if it is given for prevention. I don't say that bigger is better, I only can point out that, in the GINI study, one group grew differently, or the weight gain was lower compared to the WHO centiles. So I 
don't want to comment on which is better or worse, but to point out there are differences and they are probably due to either intake or metabolic effects.

Dr. Haschke: You pointed out, and I fully agree with you, that only a small minority of children have a proper diagnosis established before treatment. Committees make recommendations for proper diagnosis but when it comes to day-to-day practical application it is not so easy to follow complex procedures and provide the necessary logistics. To get an appointment for proper testing sometimes takes weeks, i.e. 2 weeks more with suffering. Therefore, many doctors change the diet without having established the proper diagnosis. Diagnostic tools and procedures should be simplified. How do you feel about this?

Dr. S. Koletzko: I agree, for a pediatrician in practice or a general physician it is more difficult than in a hospital setting. Many children are put on elimination diets or therapeutic formulas which, as a first step, is correct because every child should have a chance to improve whatever the symptoms are. But what is of concern is that they are kept on the diet although their symptoms don't even improve. They are not re-challenged to see whether improvement was by chance or had something to do with the elimination. We could see that in the GINI study where we were forced to do challenges. I think there is no excuse for not following this elimination challenge procedure, especially in children with unspecific symptoms like skin or GI symptoms. I think if you have angioedema it is much easier. The access for appointments may differ from country to country. I have no answer; I can't solve the logistic problems of the health systems in the different countries. I think we all should put an effort into following this simple strategy in order to avoid unnecessary diets.

Dr. Haschke: If the proper diagnosis cannot be established on time, Neocate ${ }^{\circledR}$ as an amino acid-based solution has been promoted ('try it, it will help you'). The company marketing Neocate is aggressively stepping in to grab the field before a proper diagnosis can be established.

Dr. S. Koletzko: I agree with you completely that we must try to counteract this aggressive marketing of products. I think trying something might be alright for a few days, but then you need a physician to do the proper workup, and everything else should not happen.

Dr. Venter: You showed that in the GINI study the children on extensively hydrolyzed formulas had less growth compared to the other formulas. Did you also look at volumes taken? Did they grow less because they actually drank less of the formula compared to the others?

Dr. S. Koletzko: No, the length of formula feeding was the same; it was body weight gain which was different. No we did not measure the volumes so we don't know whether the intake was less because perhaps of poor taste or other reasons. We were unable to control for that in our protocol.

Dr. Kneepkens: Thank you for a very clear overview of the pros and cons of food elimination. You very clearly showed the negative effects as well. I think we both agree that it is very important for the diagnosis to be made as accurately as possible. Therefore I wonder why you would accept open challenges as proof of food allergy. There are studies from the Netherlands showing that only one third of the children who tested positive with open challenges were proven to really have cow's milk allergy when they underwent double-blind provocation.

Dr. S. Koletzko: In infants this is for practical reasons, otherwise the availabilities of health facilities are even more complicated. If you do an open challenge and the child is tolerating then you don't need a blind one, but if you have a reaction and if there is doubt, e.g. a flair up of skin or eczema, then this open challenge should be followed by a double-blind. But I think to ask for a double-blind as first line for all children is just not feasible. 
Dr. Kneepkens: I agree with you that when an open challenge doesn't provoke any reaction, a double-blind challenge is not necessary, but I think that all children with a positive open challenge, even if the challenge seems to be clearly positive, should be double-blindly challenged. Otherwise too many children will be prescribed elimination diets.

Dr. S. Koletzko: I think again you have to balance because double-blind challenge needs to be done in hospital, the other can be on an outpatient basis. I think we have to be aware of the problem and have to make individual decisions, but we have to be aware that open challenge can be false-positive. I agree with you that in older children we should aim for double-blind challenges.

Dr. Isolauri: One comment to this specific question. We made a study where we randomized infants to open and double-blind challenges and in the unselected group randomly assigned to one of these two groups there were no differences in the rate of positive reactions in infancy. I also think infancy is a critical period to introduce new foods. Therefore, accurate diagnosis is crucial to promote age-appropriate diets also in the allergic infant. So this is a very practical early treatment rather than repeated challenges.

Dr. S. Koletzko: No, I don't agree. I think if an infant on a therapeutic diet who improves after elimination of cow's milk formula should be challenged within a decent time, 2-4 weeks and not kept for months, or did I misunderstand you?

Dr. Isolauri: I said that once you have the diagnosis of cow's milk allergy, concentrate on treating the child and put some age-appropriate solid foods in the diet and then repeat the challenge after 6 months, either openly or in a blinded manner.

Dr. S. Koletzko: I agree, and this is actually according to the algorithms which can be easily done in an outpatient setting in the vast majority of the infants.

Dr. Berdel: There are also hydrolyzed soy formulas on the market. Are there the same recommendations and the same restrictions?

Dr. S. Koletzko: It is not clear how much phytate and isoflavones are contained in hydrolyzed soy protein. It is a mixture of hydrolyzed collagen and soy. Based on cow's milk protein the isoflavone and phytate contents are of course higher in this hydrolyzed formula. The restrictions also depend on the level, whether it is 20 or $80 \%$. But it is not clear how much there is in there and I think it should be declared.

Dr. von Berg: After diagnosis and elimination or putting the child on a hydrolysate, at what time would you do the re-challenge?

Dr. S. Koletzko: It depends on the organ manifestation. If there is an acute reaction and a positive challenge result, at some point specific IgE will be measured, and if it is positive I would wait at least 6 months or at least after the age of 1 year, and then I would repeat it. It is different in children who have bloody mucous diarrhea with and without breastfeeding or cow's milk feeding, what we call allergic colitis. There are good studies showing that this is a more benign or self-limiting disease. Many of these children are put on a therapeutic diet but may not even have cow's milk allergy. In these children we re-challenge much earlier, after 3 months or so or when they have supplementary feeding, and many of them tolerate cow's milk. So it depends on the manifestation and whether they were IgE-positive or not.

Dr. Baerlocher: Relating to the study you showed from Utrecht where a stronger reaction was found following the accidental intake of allergens, I thought this was an old clinical allergological experience. I have seen this several times in children who were on a strong diet and had an accidental intake, the reaction was much stronger.

Dr. S. Koletzko: The difference is that these 11 children were selected from an outpatient clinic and had never had a reaction before. They were put on the diet without reaction and without improvement, but were kept on the diet. So certainly these 
were highly allergic children. But still the question remains, would the outcome have been different if they had been kept on cow's milk protein early in life? Nobody knows the answer. What you said is correct. We know that following prolonged elimination, an acute reaction can be even more severe in accidental ingestion. The observations point to what we heard previously that continuous small amounts may induce tolerance rather than strict elimination, and I think this needs to be evaluated.

Dr. Szajewska: I have a question related to the guidelines regarding treatment which you published some time ago [1]. When I read them I had the impression that there was a lot of emphasis on the elemental diet, and as Dr. Haschke mentioned, there was also a lot of attention paid to this diet during the World Congress. Here as I listened to your lecture, I had the impression that you are not very much in favor of this treatment anymore. Could you comment on the place of the elemental diet in the treatment of cow's milk allergy? My second question is, if it were not for the price, which is very high in many countries, would you have started treatment with the elemental diet and then gone over to an extensively hydrolyzed formula?

Dr. S. Koletzko: With respect to this publication, first of all it is clearly stated that in all children with mild to moderate manifestations, whatever organ, hydrolyzed formula is first choice. Only in children who do not tolerate or do not improve or may refuse to drink, you can give a trial of amino acid. The exception is stated in the paper, although it is not evidence-based, it is practical in children with severe reactions, acute life-threatening reactions, that you should not take the risk of $10 \%$ not tolerating the hydrolyzed formula, and they should be put directly on amino acid just to be on the safe side. Actually that is not that much different from what I said today. I want to point out that several mistakes were made in this original publication. Especially the algorithm for formula-fed infants was wrong. We have asked to have this article reprinted with the correct figure.

Dr. Allen: Regarding the Australian guidelines for the use of soy in the over 6-month-old group; in Australia we are not as keen as the European and US guidelines to discard the use of soy in the over 6-month age group because soy formula is not the sole food. There appears to be a low risk of allergy, only $10 \%$ of the children are likely to be allergic to soy, and we don't really know the risk for the non-IgE-mediated group as we do for IgE-mediated group. About $90 \%$ of children with cow's milk allergy tolerate soy, so that's a large proportion. There is also the question of whether we are medicalizing a condition where in Australia the child maternal health nurses will often put children onto soy in the interim before they get to a doctor and the children improve. So my question is, in Europe are you seeing a change in referral patterns of children with cow's milk allergy and an increase in referrals to specialists? In Australia we have a significant problem with waiting lists at our allergy clinics.

Dr. S. Koletzko: For a child on exclusive formula feeding, the reasons for not choosing soy milk in the first half year are the high development or high risk of soy allergy, and all the other associated disadvantages. I think putting a child on soy milk while waiting for an appointment with a specialist is even worse because the sensitized child may have a gut lesion. One option could be that the general physician puts the child on an extensively hydrolyzed formula to await the specialist appointment. But this differs from country to country. Perhaps it is a health source problem, and it is very different in various countries even within Europe. In some countries these children are cared for by specialists, and in some countries they are cared for by pediatricians, unless they have complications.

Dr. Allen: My point is that we may not be seeing these children in the clinics if they have already been treated with soy because soy is not a medicalized formula, it is an over-the-counter formula in Australia. We think there are as many as $2-4 \%$ of children with cow's milk allergy and many of them are just being managed differently. 


\section{Koletzko/Koletzko}

Dr. S. Koletzko: But then you may advise that soy formula is really not a good choice in the first year of life. If you make a strong point to all pediatricians regardless of allergy, this may help you get the referrals before they are put on soy.

Dr. Allen: We are actually suggesting that in the first 6 months soy should be avoided, but by 6-12 months it is alright to use soy.

Dr. Heine: Just a comment on the need for diagnostic food challenges. It is important to distinguish between IgE-mediated and non-IgE-mediated cow's milk allergy. In the IgE-mediated form, skin testing can often help us avoid food challenges. Difficulties often arise in the non-IgE-mediated GI forms. Challenges in these patients should be performed after nutritional stabilization has been achieved on an elimination diet, but not while the child is still in a phase of catch-up growth. This could otherwise induce adverse feeding behaviors. Once the nutritional and behavioral effects of GI food allergy have settled, gradual normalization of the diet can be attempted.

Dr. S. Koletzko: That is what we have written in the guidelines. They should be kept on diagnostic therapy until they have stabilized and are no longer in a catabolic state.

\section{References}

$>1$ Vandenplas Y, Koletzko S, Isolauri E, et al: Guidelines for the diagnosis and management of cow's milk protein allergy in infants. Arch Dis Child 2007;92:902-908. 